\title{
Adsorption of fluoroquinolone antibiotics at the gas-liquid interface using ionic surfactants
}

\author{
Rajesh Ghosh ${ }^{1}$, Haritha Hareendran ${ }^{2}$, Pushpavanam Subramaniam ${ }^{1 *}$ \\ ${ }^{1}$ Department of Chemical Engineering, Indian Institute of Technology, Madras, Chennai, \\ 600036, India \\ ${ }^{2}$ Department of Chemical Engineering, Alagappa College of Technology, Anna University, \\ Chennai, 600025, India \\ *Email: spush@iitm.ac.in
}

\section{Supplementary Information}

\section{Foam fractionation using CTAB}

\subsection{Effect of $\mathbf{p H}$}

To determine the role of $\mathrm{pH}$ on the CTAB system, a surfactant concentration of $0.27 \mathrm{mM}$, initial ciprofloxacin concertation of $25.9 \mu \mathrm{M}$ and air flow rate of $25 \mathrm{~mL} / \mathrm{min}$ was maintained. Figure S1 summarizes the change in enrichment ratio, recovery ratio, volumetric separation factor and residual factor with change in $\mathrm{pH}$ of the feed solution. In this case, an inverse behavior was observed. Both enrichment ratio and recovery ratio increased with the increase in $\mathrm{pH}$ of the feed. On changing the $\mathrm{pH}$ of the feeding solution from 3.0 to 9.0, the enrichment ratio increased from 1.2 to 2.4 and the recovery ratio increased from $20.7 \%$ to $52.2 \%$. The enrichment ratio and removal ratio of ciprofloxacin obtained using $\mathrm{CTAB}$ is not as high as that using SDS, which confirms that the adsorption of the cationic surfactant and ciprofloxacin complex was not as spontaneous as that of the anionic surfactant bound ciprofloxacin. At $\mathrm{pH}$ 9, the carboxyl functional group of ciprofloxacin is deprotonated which increased the binding efficiency, promoting increased adsorption of the drug at the gas-liquid interface. The low enrichment ratio obtained could be attributed to the high liquid flux in the foam, due to the high foaming ability of CTAB. In addition, with increase in the $\mathrm{pH}$ from 3 to 9 , the volumetric separation factor changed marginally from 0.23 to 0.30 , suggesting that the foaming behavior and liquid flux in the foam is not influenced with 
change in $\mathrm{pH}$ for the $\mathrm{CTAB}$ system. The residual factor decreases with increase in $\mathrm{pH}$, indicating that more of ciprofloxacin molecules were removed at basic $\mathrm{pH}$ conditions.
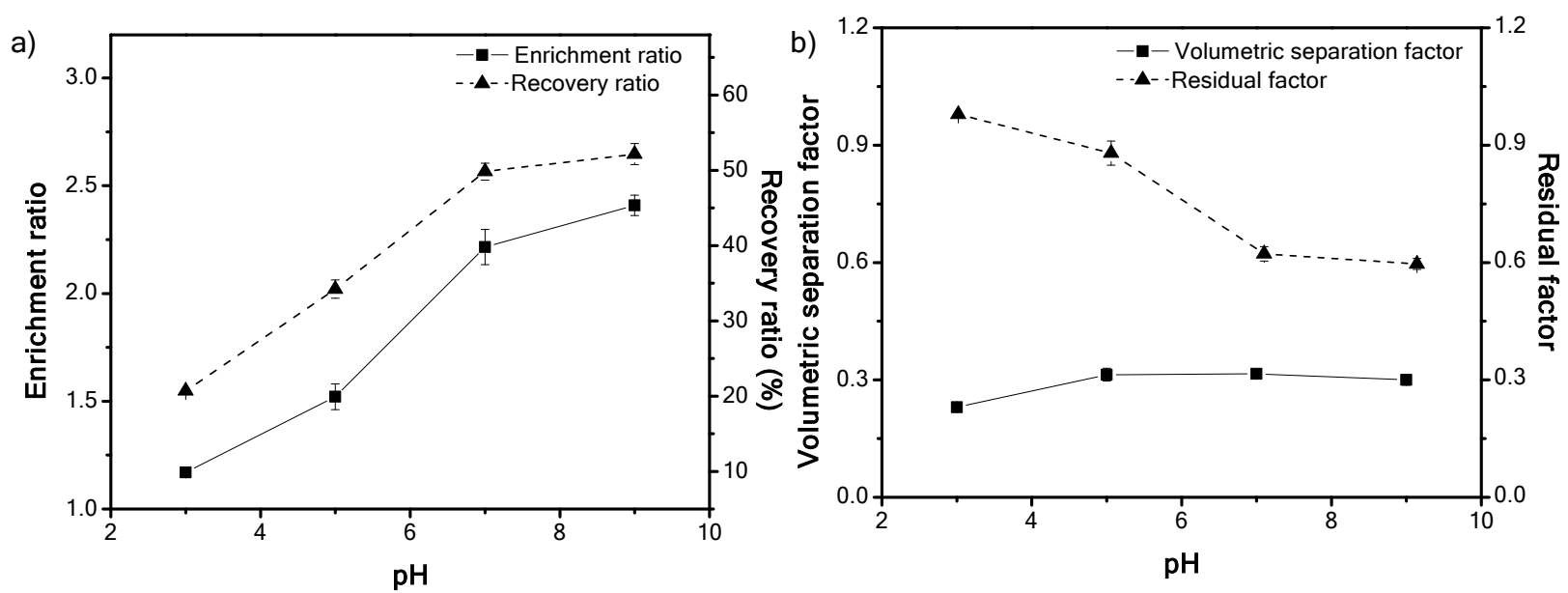

Figure S1. Effect of solution $\mathrm{pH}$ on (a) enrichment ratio (- - -) and recovery ratio (- $\left.\mathbf{\Delta}_{-}\right)$(b) volumetric separation factor $(-\mathbf{-}-)$ and residual factor $\left(-\mathbf{\Lambda}_{-}\right)$.

\subsection{Effect of CTAB concentration}

In the CTAB system, the surfactant concentration was varied from 0.05 to $0.41 \mathrm{mM}$, with constant ciprofloxacin concentration of $25.9 \mu \mathrm{M}$ and air flow rate of $25 \mathrm{~mL} / \mathrm{min}$. The $\mathrm{pH}$ of the bulk solution was maintained at 9 for the CTAB system as ciprofloxacin showed maximum binding interaction at this $\mathrm{pH}$ value. Figure $\mathrm{S} 2$ shows the variation of enrichment ratio, recovery ratio, volumetric separation factor and residual factor with change in bulk CTAB concentration. The enrichment ratio shows similar trend as that of the SDS system. A maximum enrichment of 7.6 was obtained when $\mathrm{CTAB}$ concentration in the feed solution was $0.1 \mathrm{mM}$. The recovery ratio increased with the increase in surfactant concentration. The residual factor does not show any significant decline with increase in surfactant concentration which indicates that the equilibrium of separation was reached and further increase of surfactant dosage does not positively impact the separation. The volumetric separation factor increased with surfactant concentration as expected. 

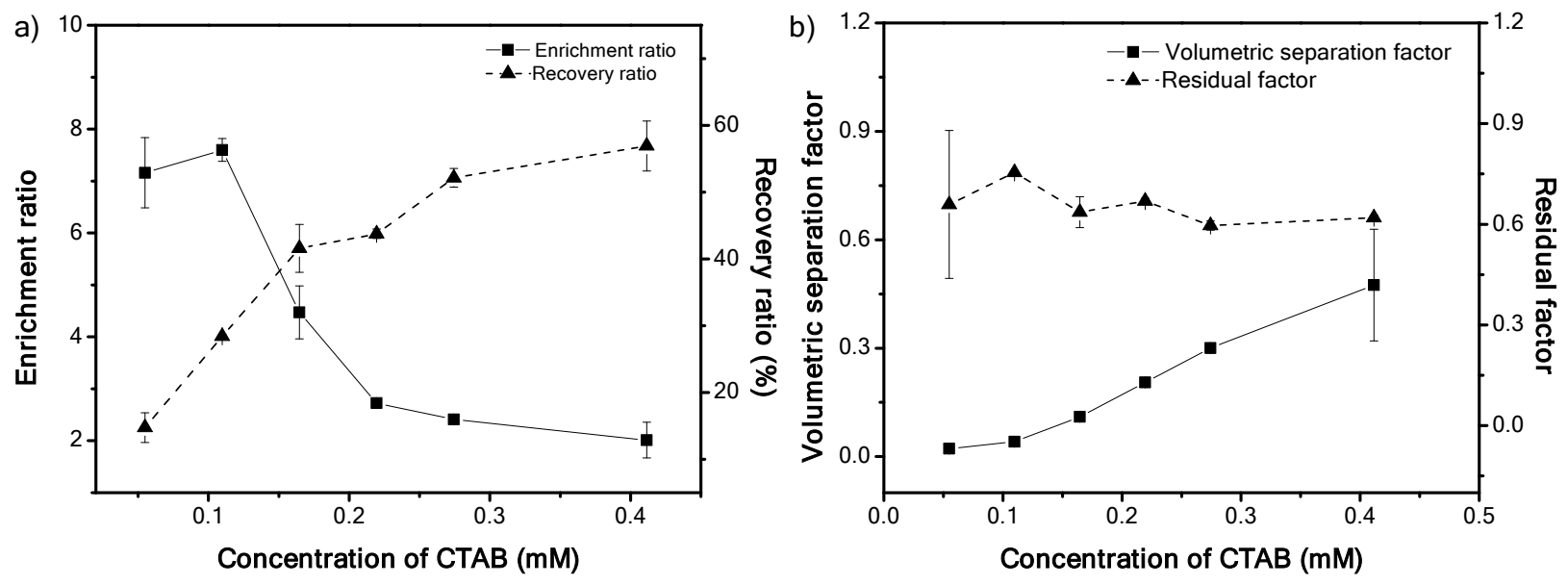

Figure S2. Effect of CTAB concentration on (a) enrichment ratio (- - -) and recovery ratio (- $\left.\mathbf{\Delta}_{-}\right)$ (b) volumetric separation factor (--) and residual factor (- $\left.\mathbf{\Delta}_{-}\right)$.

\subsection{Effect of ciprofloxacin concentration}

For the CTAB system, the initial ciprofloxacin concentration was varied from $2.5 \mu \mathrm{M}$ to $52 \mu \mathrm{M}$, with surfactant concentration fixed at $0.27 \mathrm{mM}$, gas flow rate of $25 \mathrm{~mL} / \mathrm{min}$, and solution $\mathrm{pH}$ of 9 . Figure S3 shows the enrichment ratio, recovery ratio, volumetric separation factor and residual factor for varying concentration of ciprofloxacin in the feeding solution. Both enrichment ratio and recovery ratio decrease with the increase in initial ciprofloxacin concentration. The volumetric separation factor did not show any change with change in initial ciprofloxacin concentration. On the other hand, the residue factor increased with increase in initial ciprofloxacin concentration indicating that more of the added ciprofloxacin remained in the bulk.
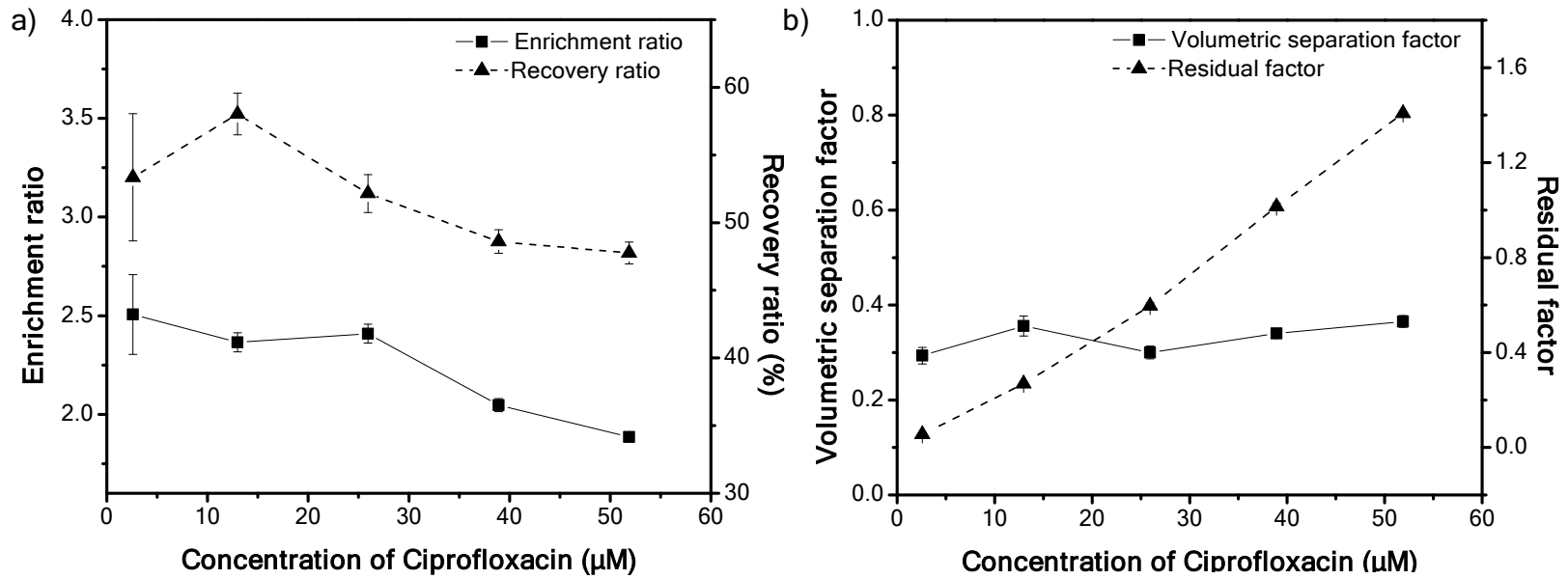

Figure S3. Effect of initial ciprofloxacin concentration on (a) enrichment ratio (- - -) and recovery ratio (- $\left.\boldsymbol{\Lambda}_{-}\right)$(b) volumetric separation factor (- -$)$and residual factor (- $\left.\boldsymbol{\Lambda}_{-}\right)$. 